\title{
Assessment of the relationship between bacteriological quality of dug-wells, hygiene behaviour and well characteristics in two cholera endemic localities in Douala, Cameroon
}

\author{
Jane-Francis Tatah Kihla Akoachere ${ }^{1,2^{*}}$, Lundi-Anne Omam ${ }^{1}$ and Thomas Njinuwo Massalla²
}

\begin{abstract}
Background: Access to potable water is grossly inadequate in Douala-Cameroon. The situation is worse in slum areas, compelling inhabitants to obtain water from sources of doubtful quality. This has contributed to frequent outbreaks of water-borne diseases particularly cholera, which results in severe morbidity and mortality. Shallow wells are a major source of water in these areas. We analyzed the influence of some factors on the bacteriological quality of well water in Bepanda and New Bell, cholera endemic localities in Douala to generate data that would serve as basis for strengthening of water and health policies.
\end{abstract}

Methods: Questionnaires were administered to inhabitants of study sites to appraise their hygiene and sanitation practices, and level of awareness of waterborne diseases. The bacteriological quality of water was determined by investigating bacterial indicators of water quality. Relationship between well characteristics and bacteriological quality of water was determined using $x^{2}$ test. The Kendall tau_b nonparametric correlation was used to measure the strength of association between well characteristics and bacteriological parameters. Statistics were discussed at $95 \%$ confidence level. Antibiotic susceptibility of isolates was investigated by the Kirby-Bauer and broth dilution techniques. Multidrug resistant species were tested for extended $\beta$-lactamase production potential.

Results: Inhabitants demonstrated adequate knowledge of waterborne diseases but employed inappropriate method (table salt) for well disinfection. Well construction and location violated guidelines. Indicator bacterial counts greatly exceeded the WHO guidelines. Variation in bacteriologic parameters between sites was not significant $(P>0.05)$ since well characteristics and hygiene and sanitary practices were similar. Differences in bacteriologic quality with respect to state of well, and presence of molded casing and lid, and height of casing were not significant $(P>0.05)$. Well distance from sanitary structure negatively correlated with bacteriological characteristics indicating it could be a major contributory factor to poor water quality. Bacteria isolated were predominantly enteric organisms. Ciprofloxacin was the most active agent. Extended $\beta$-lactamase producers were detected among Salmonella species, Citrobacter fruendii and E. coli.

Conclusion: Poor well location, construction, and hygiene and sanitary practices were among the factors affecting water quality. There is an urgent need for education of inhabitants on effective water disinfection strategies and for regular monitoring of wells.

Keywords: Dug-well characteristics, Water quality, Sanitation, Hygiene, Bacterial pathogens, Antibiotic susceptibility, Waterborne diseases, Extended $\beta$-lactamase producers

\footnotetext{
* Correspondence: jakoachere@yahoo.com

'Department of Microbiology and Parasitology, Faculty of Science, University of Buea, Buea, Cameroon

2Laboratory for Emerging Infectious Diseases, Faculty of Science, University of Buea, Buea, Cameroon
} 


\section{Background}

Water is a necessity for life. The ability to provide potable water thus affects health, ecosystems and the economy. Although many countries of the world are investing enormous resources to meet the growing demand for good quality water, the supply of potable water is still a great challenge particularly in developing countries where urbanization, industrialization and rapid population growth in the context of limited financial resources has placed a burden on water resources [1]. In these countries, lack or inadequate supply of potable water in addition to absence of basic sanitary and hygiene practices has resulted in increased morbidity and mortality from waterborne diseases particularly among children less than 5 years old [2]. In areas facing severe scarcity ground water is used as the main source of water for drinking and other purposes. Due to poverty and lack of technology to construct deep wells, majority of the population in developing countries obtain ground water from shallow wells which are easy and cheaper to construct. Unfortunately, overcrowding, poor hygiene and sanitation have subjected water in these wells to contamination with pathogenic organisms making them a potential source of health hazard.

Access to potable water in both rural and urban centers of Cameroon is a great concern [3,4]. Studies carried out in different parts of the country [5-7] show that most domestic water sources have disturbing levels of microbial pollution and this has increased the prevalence of waterborne diseases. Water related diseases have been estimated to account for two-thirds of diseases and for about $50 \%$ of deaths in Cameroon [8]. Because of this, water and sanitation has been identified by the government as one of the major pillars of the long term development goals in becoming an emerging economy by the year 2035. Therefore, studies on water quality and water conservation are of prime importance in providing data that will facilitate the government's efforts not only in becoming an emerging economy but also to meet Target 7C of the Millennium Development Goals [9] and to improve the quality of life of Cameroonians.

In Douala, the economic capital and main port city of Cameroon, only about 650,000 persons out of a population of over 3 million [10] have access to potable water. Less than 20 million $\mathrm{m}^{3}$ of water is distributed annually by "Camerounaise Des Eaux" (CDE), the only company that distributes potable water in Cameroon. In parts of the city with potable water, supply is intermittent. The problem of potable water scarcity is more severe in slum areas of the city. To meet their water needs inhabitants obtain water from underground sources. Since the water table is high, hand-dug wells have served as an easier way to access ground water. Unfortunately, the quality of water from hand-dug wells is generally poor as confirmed by the regular outbreaks of waterborne diseases such as cholera which always begin from slum areas [8]. Thus frequent evaluation of well water quality and identification of potential sources of hazard is highly necessary in Douala to formulate policies and design strategies to improve water quality.

Our study was aimed at assessing some factors including well characteristics, hygiene and sanitation behavior of inhabitants, affecting the bacteriological quality of well water in Bepanda and New Bell, two localities in Douala that have always served as starting points for cholera outbreaks. The susceptibility of pathogenic or potentially pathogenic bacterial isolates to antibiotics that have been extensively used in prophylaxis or treatment of waterborne diseases in Douala was also investigated to determine suitable agents for current use especially as drug resistance has been reported [11]. Findings are expected to strengthen water and health policies and facilitate Cameroon's achievement of health and water-related Millennium Development Goals (MDGs).

\section{Materials and methods Study sites}

The study was carried out in New Bell and Bepanda (Figure 1), two densely populated unplanned neighborhoods in Douala, Littoral Region. The city of Douala has a population density of over 2000 inhabitants $/ \mathrm{km}^{2}$ [12] and housing density of 3 persons/room [13] and is located between $4^{\circ} 04^{\prime}$ at latitude North and $9^{\circ} 45^{\prime}$ at longitude East, along the coastal plain of Cameroon. Douala has an average rainfall of $2900 \mathrm{~mm}$ per year with an equatorial climate of two seasons: the dry season which begins in November and ends in April, with January and February being the hottest months [14], and the rainy season begins from April to October, with August and September having the highest rainfall. Temperature variations are between $26^{\circ} \mathrm{C}$ and $31^{\circ} \mathrm{C}$. The city is poorly drained and this causes flooding during periods of heavy rains. Douala lacks a sewerage system and sewage treatment facilities hence untreated sewage is discharged indiscriminately into the environment. Although a few streams run through the study sites, dug-wells constitute the main source of water.

\section{Study design}

Inhabitants' knowledge of waterborne diseases, and their hygiene and sanitation practices was evaluated through responses to questionnaires. Dug-wells (75 each in Bepanda and New Bell) were randomly selected and their characteristics investigated. Water samples (450) were collected from these wells from February to November 2011in sterile bottles $(500 \mathrm{ml})$ and their bacteriologic quality investigated. The relationship between well characteristics and bacteriological quality of samples was analyzed statistically. The antibiotic susceptibility of the isolates was also 


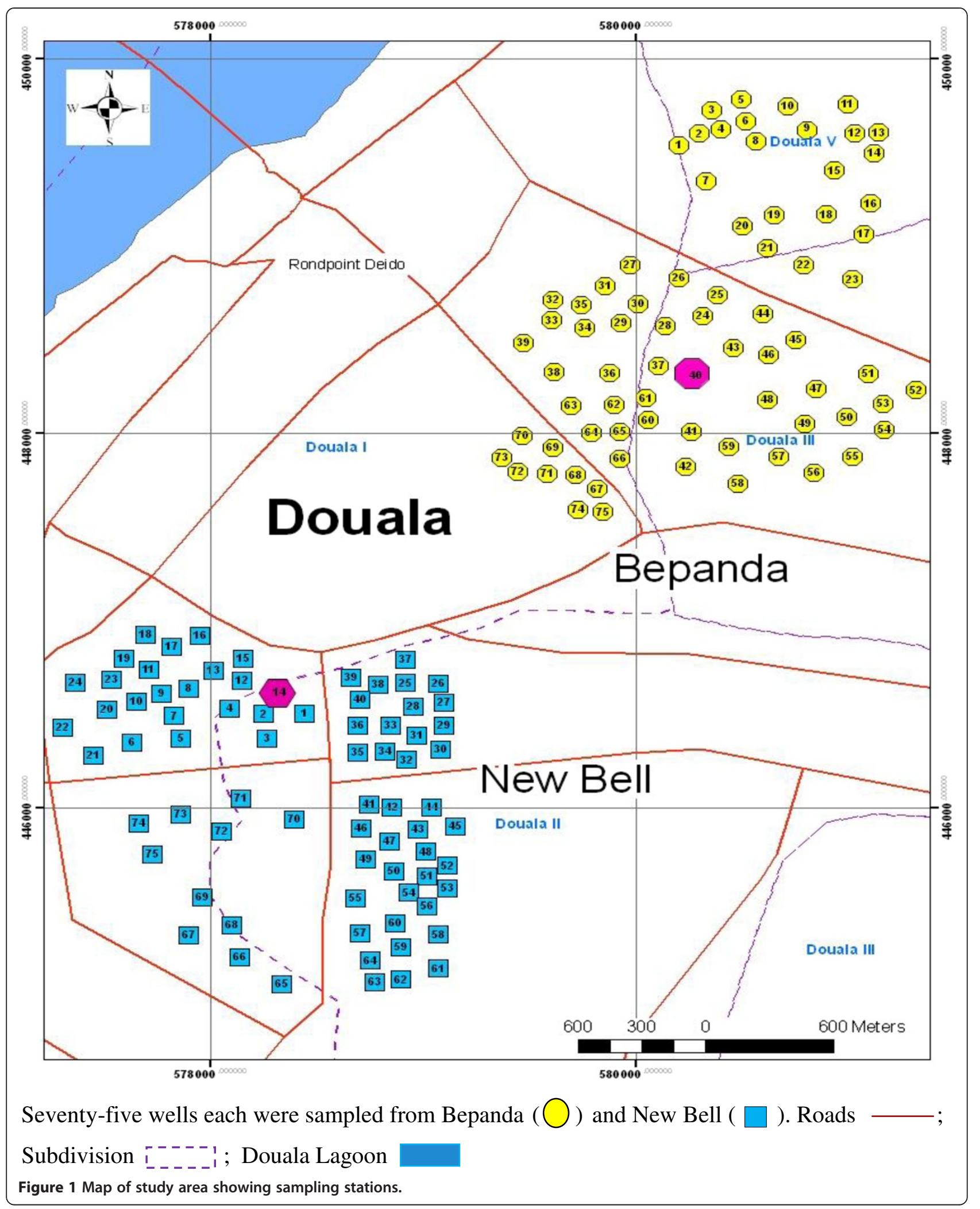


determined to ascertain effective drugs that could be used for treatment of waterborne diseases.

Ethical approval to collect data for this study was obtained from the Faculty of Health Sciences Institutional Review Board of the University of Buea (No. 2011-02 -0159). Authorization to collect water samples was obtained from the District Officers of Douala Deuxieme and Douala Troisieme where study sites are located and from well owners after a thorough explanation of the purpose of the study.

\section{Questionnaire survey}

Questionnaires (307) were administered to inhabitants of study area who use well water to obtain information on various uses of water, hygiene and sanitation practices and assess their knowledge of water borne diseases.

\section{Evaluation of well characteristics}

The distance between perimeter of well opening and sanitary structures (pit latrines or septic tanks) was measured using a Global Positioning System (Guangzhou Making Electronic Technology Co., Ltd, China). The height of well casing above ground level was determined using a measuring tape. Other characteristics of wells such as presence of molded casing, presence of lid, and condition of well (old, fairly old or new) were determined by visual inspection.

\section{Sample collection}

A string disinfected with $70 \%$ alcohol was tied around the neck of a sterile bottle. The bottle was lowered into the well without it touching the walls of the well. When full, the bottle was pulled out and immediately capped. Samples were labeled appropriately and placed in a box with ice packs and transported to the laboratory. Samples were processed within 24 hours of collection.

\section{Bacteriological analyses \\ Determination of bacterial counts}

The standard plate count method was used to determine the total viable bacterial population. Coliforms were enumerated as described by Siham and Taha [15] using violet red bile lactose (VRBL) agar by pour plate technique. Plates were inoculated in duplicate. One plate was incubated at $37^{\circ} \mathrm{C}$ for 48 hours to isolate total coliforms and the other at $44.5^{\circ} \mathrm{C}$ for 48 hours to permit the growth of fecal coliforms. Colonies developed in each plate were counted.

To determine Vibrio counts, the method described by Shanmugam and Santhanam [16] was used. One hundred microlitres of sample was inoculated onto Thiosulphate Citrate Bile Salt Sucrose (TCBS) agar by pour plate technique. Colonies developed after incubation at $37^{\circ} \mathrm{C}$ for 48 hours were counted.

\section{Isolation of bacteria}

Samples were inoculated onto nutrient agar, MacConkey agar, mannitol salt agar, eosine methylene blue (EMB) agar and Salmonella-Shigella agar, and plates incubated at $37^{\circ} \mathrm{C}$ for 24 hours. Pure cultures were subjected to gram staining, oxidase test, catalase test, motility test, coagulase test (for suspected Staphylococcus) and growth on Triple Sugar Iron (TSI) agar. Isolates were presumptively identified based on morphological, cultural and biochemical characteristics. The API 20E kit (BioMerieux SA, France) was used to confirm the identity of gram negative isolates.

To isolate Vibrio cholerae and other Vibrio species, samples were pre-enriched in alkaline peptone water (APW), $\mathrm{pH} 8.4$, at $37^{\circ} \mathrm{C}$ for 24 hours. A loopful of the surface pellicle from the enriched culture was streaked on TCBS agar and incubated at $37^{\circ} \mathrm{C}$ for 24 hours. Yellow colonies on TCBS agar measuring 2-4 mm diameter were presumptively identified as $V$. cholerae. Green and other yellow colonies were typical of other vibrios. Isolates were characterized as described above. $V$. cholerae isolates were serotyped using $V$. cholerae $\mathrm{O} 1$ and $\mathrm{O} 139$ polyvalent antisera as described by CDC [17].

\section{Antibiotic Susceptibility Testing (AST)}

The disc diffusion method was used. Antibiotic discs tested were from Liofilchem Diagnostics, Italy and included tetracyclines [tetracycline $(30 \mu \mathrm{g})$ and doxycycline $(30 \mu \mathrm{g})$ ], $\beta$ lactams [ampicillin $(10 \mu \mathrm{g})$ and ceftriaxone $(30 \mu \mathrm{g})$ ], folic acid synthesis inhibitor [cotrimoxazole $(25 \mu \mathrm{g})$ ], fluoroquinolone [ciprofloxacin $(30 \mu \mathrm{g})$ ], protein synthesis inhibitor [chloramphenicol $(30 \mu \mathrm{g})$ ] and aminoglycoside [gentamicin $(10 \mu \mathrm{g})]$.

MIC (minimum inhibitory concentration) was determined by the broth dilution assay [18]. MIC was determined only for antibiotics to which isolates showed sensitivity following testing by disc diffusion technique.

\section{Determination of extended spectrum beta-lactamase production}

Observing that all multidrug resistant isolates (resistant to 3 or more drugs) showed resistance to $\beta$-lactam antibiotics, we investigated their extended spectrum beta-lactamase (ESBL) production potential. The disc approximation method [19] was employed. An increase in the zone of inhibition due to the ceftazidime-amoxicillin-clavulanate-ceftriaxone disc synergy showed that the organism had potential for extended spectrum $\beta$-lactamase production. The diameters were compared with recommended standards, which conform to those of the Clinical Laboratory Standard Institute (CLSI) [20].

\section{Statistical analyses}

Data was analyzed using the Statistical Package for Social Sciences (SPSS) version 17.0. Basic statistics for nominal 
and ordinal variables were performed using frequencies. Measures of central tendency were employed to study numeric variables and their underlying distributions. Relationship between well characteristics and water quality was assessed using Pearson's Chi-square test. Fisher's Exact Test (FET) was used in situations where cross tabulation procedure for Chi-square produced one degree of freedom with any minimum expected count of less than 5 . The Kendall tau_b nonparametric correlation coefficient was used to measure the strength and direction of association between scale variables. The choice of this test was because our data for well casing elevation, distance of wells from sanitary structure and bacterial counts were not normally distributed. All statistics were discussed at 95\% confidence level $(\alpha, 0.05)$.

\section{Results}

\section{Responses to questionnaires}

Of the 307 questionnaires, 153 (49.8\%) were administered in Bepanda and 154 (50.2\%) in New Bell. Seventy-three percent $(73 \%)$ of the respondents were female, majority (53.7\%) of who were house wives. Most respondents had acquired at least basic education. Only one respondent $(0.33 \%)$ reported using well water for drinking (Additional file 1). The rest obtained drinking water from tube wells and communal stand pipes. Uses of well water reported included cleaning (100\%), cooking (73.3\%) and washing of fruits and vegetables (50.4\%). Inhabitants lifted well water by the bucket and rope method. One hundred and eighty two $(50.9 \%)$ respondents treated their wells though not frequently, with the majority (88.5\%) employing table salt as disinfectant (Additional file 1). A significant fraction of respondents $(76.5 \%)$ had knowledge of well water contamination and possible sources of contamination. Use of pit latrines (93.5\%) was the main method of human waste disposal. Inhabitants (64.2\%) disposed domestic waste in public vats. All respondents $(100 \%)$ had knowledge of waterborne diseases, with $73.0 \%$ having been victims.

\section{Characteristics of wells studied}

Of the 150 wells sampled, only $68(45.3 \%)$ had a lid (Table 1). The casing of nine wells $(6.0 \%)$ was not raised above the ground. Only $48 \%$ of wells had casing raised $\geq 0.6 \mathrm{~m}$ above ground level. Distances between wells and sanitary structures ranged from $1 \mathrm{~m}$ to $17.4 \mathrm{~m}$ (mean distance $=7.4 \mathrm{~m}$ and $7.6 \mathrm{~m}$ respectively for Bepanda and New Bell). One hundred and eight wells (72\%) were located within a distance of $10 \mathrm{~m}$ from a sanitary structure. Eighty-five (56.6\%) wells were in a poor state, looking very old and were poorly maintained. Overall, 79.3\% of the wells had a molded casing but many of these wells had cracks on the casing.

\section{Bacteria counts}

Bacteria counts were generally high throughout the period of study. In New Bell total viable bacterial counts (TVBC) ranged from 0.0 to $4.25 \times 10^{4} \mathrm{CFU} / \mathrm{mL}$ while in Bepanda, counts obtained varied from 0.0 to $1.5 \times 10^{4} \mathrm{CFU} / \mathrm{mL}$ (Table 2). There was no significant difference in counts between study sites $\left(\chi^{2}=1.931, d f=1, P=0.165\right)$. Counts were higher in the rainy season than in the dry season. There was a significant difference in total viable bacterial counts between seasons (FET P <0.001).

Total coliform counts ranged from 0.0 to $1.05 \times$ $10^{4} \mathrm{CFU} / \mathrm{ml}$ in Bepanda and 0.0 to $1.4 \times 10^{4} \mathrm{CFU} / \mathrm{ml}$ in New Bell (Table 2). There was no significant difference in counts between sites $\left(X^{2}=0.409\right.$, df $\left.=1, P=0.522\right)$. Very high fecal coliform counts were recorded throughout the

Table 1 Characteristics of wells studied

\begin{tabular}{|c|c|c|c|c|}
\hline \multirow[t]{2}{*}{ Characteristic } & \multirow[t]{2}{*}{ Category } & \multicolumn{2}{|c|}{ Study site } & \multirow[t]{2}{*}{ Total N (\%) } \\
\hline & & Bepanda N (\%) & New Bell N (\%) & \\
\hline \multirow[t]{2}{*}{ Aperture of well covered } & Yes & $38(25.3 \%)$ & $30(20 \%)$ & $68(45.3 \%)$ \\
\hline & No & $37(24.7 \%)$ & 45 (30\%) & $82(54.7 \%)$ \\
\hline \multirow[t]{3}{*}{ Well elevation above ground $(\mathrm{m})$} & 0.00 & $3(2.0 \%)$ & $6(4.0 \%)$ & $9(6.0 \%)$ \\
\hline & $0.1-0.59$ & $30(20.0 \%)$ & $39(26.0 \%)$ & $69(46.0 \%)$ \\
\hline & $\geq 0.60$ & $42(28.0 \%)$ & $30(20.0 \%)$ & $72(48.0 \%)$ \\
\hline \multirow[t]{3}{*}{ Distance between well and sanitary structure $(\mathrm{m})$} & $0.0-10.00$ & $57(38 \%)$ & $51(34 \%)$ & $108(72 \%)$ \\
\hline & $10.1-15.00$ & $17(11.3 \%)$ & $19(12.7 \%)$ & $36(24 \%)$ \\
\hline & $\geq 15.00$ & $1(0.7 \%)$ & $5(3.3 \%)$ & $6(4 \%)$ \\
\hline \multirow[t]{3}{*}{ General state of well } & Old & $44(29.3 \%$ & $41(27.3 \%)$ & $85(56.6 \%)$ \\
\hline & Fairly old & $26(17.35 \%)$ & $29(19.3 \%)$ & $55(36.7 \%)$ \\
\hline & New & $5(3.3 \%)$ & $5(30.3 \%)$ & $10(6.6 \%)$ \\
\hline \multirow[t]{2}{*}{ Presence of molded casing } & Yes & $61(81.3 \%)$ & $58(77.3)$ & $119(79.3 \%)$ \\
\hline & No & $14(18.7 \%)$ & $17(22.7 \%)$ & $31(20.7 \%)$ \\
\hline
\end{tabular}


Table 2 Bacterial population of samples with respect to site and season

\begin{tabular}{|c|c|c|c|c|c|c|}
\hline Characteristic & Study site & Season & Mean counts (CFU/ml) & SE Mean & StDev & Range (CFU/ml) \\
\hline \multirow[t]{6}{*}{ Heterotrophic Bacteria Counts } & Bepanda & Dry & $2.28 \times 10^{3}$ & 271 & 3068 & $0-1.35 \times 10^{4}$ \\
\hline & & Rainy & $4.93 \times 10^{3}$ & 471 & 4638 & $0-1.5 \times 10^{4}$ \\
\hline & New Bell & Dry & $2.82 \times 10^{3}$ & 385 & 3554 & $0-1.77 \times 10^{4}$ \\
\hline & & Rainy & $5.46 \times 10^{3}$ & 471 & 5571 & $4.0 \times 10^{1}-4.25 \times 10^{4}$ \\
\hline & Total & Dry & $2.5 \times 10^{3}$ & 224 & 3273 & $0-1.77 \times 10^{4}$ \\
\hline & & Rainy & $5.252 \times 10^{3}$ & 338 & 5206 & $0-4.25 \times 10^{4}$ \\
\hline \multirow[t]{6}{*}{ Total Coliform Counts } & Bepanda & Dry & $1.12 \times 10^{3}$ & 139 & 1567 & $0-7.5 \times 10^{3}$ \\
\hline & & Rainy & $1.84 \times 10^{3}$ & 229 & 2256 & $0-1.05 \times 10^{4}$ \\
\hline & New Bell & Dry & $1.87 \times 10^{3}$ & 253 & 2329 & $0-9.8 \times 10^{3}$ \\
\hline & & Rainy & $3.20 \times 10^{3}$ & 275 & 3259 & $2.0 \times 10^{1}-1.4 \times 10^{4}$ \\
\hline & Total & Dry & $1.42 \times 10^{3}$ & 133 & 1938 & $0-9.8 \times 10^{3}$ \\
\hline & & Rainy & $2.64 \times 10^{3}$ & 192 & 2963 & $0-1.4 \times 10^{4}$ \\
\hline \multirow[t]{6}{*}{ Fecal Coliform Counts } & Bepanda & Dry & $5.89 \times 10^{2}$ & 96 & 1087 & $0-7.0 \times 10^{3}$ \\
\hline & & Rainy & $1.14 \times 10^{3}$ & 170 & 1677 & $0-9.0 \times 10^{3}$ \\
\hline & New Bell & Dry & $6.25 \times 10^{2}$ & 122 & 1124 & $0-6.0 \times 10^{3}$ \\
\hline & & Rainy & $2.05 \times 10^{3}$ & 195 & 2309 & $1.0 \times 10^{1}-9.0 \times 10^{3}$ \\
\hline & Total & Dry & $6.03 \times 10^{2}$ & 75 & 1099 & $0-7.0 \times 10^{3}$ \\
\hline & & Rainy & $1.68 \times 10^{3}$ & 138 & 2119 & $0-9.0 \times 10^{3}$ \\
\hline \multirow[t]{6}{*}{ Total Vibrio Counts } & Bepanda & Dry & $4.96 \times 10^{2}$ & 79 & 891 & $0-7.0 \times 10^{3}$ \\
\hline & & Rainy & $8.14 \times 10^{2}$ & 105 & 1038 & $0-5.0 \times 10^{3}$ \\
\hline & New Bell & Dry & $7.26 \times 10^{3}$ & 141 & 1302 & $0-7.2 \times 10^{3}$ \\
\hline & & Rainy & $1.24 \times 10^{3}$ & 122 & 1448 & $2.0 \times 10^{1}-8.0 \times 10^{3}$ \\
\hline & Total & Dry & $5.88 \times 10^{3}$ & 74 & 1077 & $0-7.2 \times 10^{3}$ \\
\hline & & Rainy & $1.06 \times 10^{3}$ & 85 & 1311 & $0-8.0 \times 10^{3}$ \\
\hline
\end{tabular}

SE mean Standard Error of mean, StDev Standard Deviation.

study (Table 2). In Bepanda, mean fecal coliform counts obtained were $5.89 \times 10^{2} \mathrm{CFU} / \mathrm{mL}$ and $1.14 \times 10^{3} \mathrm{CFU} /$ $\mathrm{mL}$ respectively for the dry and rainy seasons. Mean counts recorded in New Bell were $6.03 \times 10^{2} \mathrm{CFU} / \mathrm{mL}$ in the dry season and $2.05 \times 10^{3} \mathrm{CFU} / \mathrm{mL}$ during the rainy season. Although indicator bacterial counts were generally higher in the rainy season than in the dry season, there was no significant difference in total coliform counts (FET $\mathrm{P}=0.052$ ) with respect to season. However, significant differences were observed in fecal coliform $($ FET $\mathrm{P}=0.001$ ) count with season.

Vibrios were detected in samples throughout the period of study with counts ranging from 0.0 to $8.0 \times 10^{3} \mathrm{CFU} /$ $\mathrm{mL}$. Higher counts occurred in New Bell $(0.0$ to $8.0 \times$ $\left.10^{3} \mathrm{CFU} / \mathrm{mL}\right)$ than in Bepanda $\left(0.0\right.$ to $\left.7.0 \times 10^{3} \mathrm{CFU} / \mathrm{mL}\right)$ but the difference was not significant $\left(\chi^{2}=1.931, \mathrm{df}=1\right.$, $\mathrm{P}=0.161)$. Counts recorded in the dry season were significantly lower $\left(\mathrm{X}^{2}=16.508, \mathrm{df}=1, \mathrm{P}=0.000\right)$ than rainy season counts.

A strong positive correlation occurred between fecal coliforms and total coliforms $(+0.635)$ while correlations between fecal coliforms and TVBC $(+0.468)$, and fecal coliforms and Vibrio counts $(+0.433)$ though positive were weaker.

\section{Relationship between well characteristics and bacteriological quality of water}

TVBC were higher in wells without a lid (mean $=4.73 \times$ $10^{3} \mathrm{CFU} / \mathrm{mL}$ ) than in wells with a cover (mean $=3.73 \times$ $10^{3} \mathrm{CFU} / \mathrm{mL}$ ) (Table 3). The difference was not significant $\left(\mathrm{X}^{2}=0.400, \mathrm{df}=1, \mathrm{P}=0.527\right)$. The lowest mean TVBC $\left(3.80 \times 10^{3} \mathrm{CFU} / \mathrm{mL}\right)$ was recorded in wells with casing elevation between $0.1 \mathrm{~m}-0.59 \mathrm{~m}$. There was no significant difference in TVBC with respect to height of well casing $\left(\mathrm{X}^{2}=0.868, \mathrm{df}=2, \mathrm{P}=0.648\right)$ (Table 3$)$. A negative correlation $(-0.003)$ was observed between well elevation and TVBC. Total viable bacterial counts decreased with increase in distance from sanitary structure (Table 3 ). The lowest mean count of $2.64 \times 10^{3} \mathrm{CFU} / \mathrm{mL}$ occurred in wells located at a distance greater than $15 \mathrm{~m}$ from sanitary structure. However, there was no significant difference $\left(X^{2}=0.750, \mathrm{df}=2, \mathrm{P}=0.687\right)$ in counts with respect to distance from a sanitary structure. The correlation between well distance from sanitary structure 
Table 3 Relationship between well characteristics and heterotrophic bacterial load of samples

\begin{tabular}{|c|c|c|c|c|c|c|}
\hline Well characteristic & Category & $\begin{array}{l}\text { Mean counts } \\
\text { (CFU/ml) }\end{array}$ & SE Mean & StDev & $\begin{array}{l}\text { Range } \\
\text { (CFU/ml) }\end{array}$ & $\begin{array}{l}\text { Correlation coefficient } \\
\text { ( } P \text { value) }\end{array}$ \\
\hline \multirow[t]{2}{*}{ Condition of well aperture } & Covered & $3.73 \times 10^{3}$ & 298 & 4253 & $0-2.5 \times 10^{4}$ & \\
\hline & Not covered & $4.13 \times 10^{3}$ & 311 & 4876 & $0-4.25 \times 10^{4}$ & \\
\hline \multirow[t]{3}{*}{ Height of well casing above the ground $(m)$} & 0.00 & $4.67 \times 10^{3}$ & 933 & 4847 & $0-1.77 \times 10^{4}$ & $-0.003(0.921)$ \\
\hline & $0.1-0.59$ & $3.80 \times 10^{3}$ & 335 & 4819 & $0-4.25 \times 10^{4}$ & \\
\hline & $\geq 0.60$ & $4.00 \times 10^{3}$ & 297 & 4367 & $0-2.50 \times 10^{4}$ & \\
\hline \multirow[t]{3}{*}{ Distance between well and sanitary structure $(\mathrm{m})$} & $1.0-10.00$ & $4.13 \times 10^{3}$ & 266 & 4784 & $0-4.25 \times 10^{4}$ & $-0.119(0.000)$ \\
\hline & $10.1-15.00$ & $3.61 \times 10^{3}$ & 402 & 4182 & $0-1.77 \times 10^{4}$ & \\
\hline & $\geq 15.00$ & $2.64 \times 10^{3}$ & 801 & 3399 & $2.6 \times 10^{4}-1.05 \times 10^{4}$ & \\
\hline \multirow[t]{3}{*}{ State of well } & New & $3.56 \times 10^{3}$ & 808 & 4199 & $1.34 \times 10^{4}$ & \\
\hline & Fairly old & $3.38 \times 10^{3}$ & 358 & 4161 & $4.0 \times 10^{1}-2.5 \times 10^{4}$ & \\
\hline & Old & $4.27 \times 10^{3}$ & 286 & 4834 & $4.25 \times 10^{4}$ & \\
\hline \multirow[t]{2}{*}{ Presence of molded casing } & No & $4.08 \times 10^{3}$ & 427 & 4052 & $0-4.25 \times 10^{4}$ & \\
\hline & Yes & $3.932 \times 10^{3}$ & 251 & 4750 & $2.50 \times 10^{4}$ & \\
\hline
\end{tabular}

Well aperture $\left(X^{2}=0.400, d f=1, P=0.527\right)$. Well height $\left(X^{2}=0.868, d f=2, P=0.648\right)$.

Distance well-pit latrine $\left(x^{2}=0.750, d f=2, P=0.687\right)$. State of well $\left(X^{2}=3.486, d f=2, P=0.175\right)$. Well casing $\left(x^{2}=0.697, d f=1, P=0.697\right)$.

and TVBC was negative $(-0.119)$. Highest mean TVBC occurred in old wells $\left(4.27 \times 10^{3} \mathrm{CFU} / \mathrm{mL}\right)$ (Table 3$)$. There was no significant difference in counts with respect to the state of wells $\left(X^{2}=3.486, \mathrm{df}=2, \mathrm{P}=0.175\right)$. Wells without a molded casing had higher TVBC (mean $\left.=4.08 \times 10^{3} \mathrm{CFU} / \mathrm{mL}\right)$ compared to wells with molded casing $\left(\right.$ mean $\left.=3.932 \times 10^{3} \mathrm{CFU} / \mathrm{mL}\right)$ (Table 3$)$. The difference was not significant $\left(\chi^{2}=0.697, \mathrm{df}=1\right.$, $\mathrm{P}=0.697$ ).

Total coliform counts were higher in open wells (mean $\left.=2.14 \times 10^{3} \mathrm{CFU} / \mathrm{mL}\right)$ than in wells with cover $\left(\right.$ mean $\left.=1.98 \times 10^{3} \mathrm{CFU} / \mathrm{mL}\right) \quad($ Table 4$)$. The difference was not significant $\left(\chi^{2}=0.888, \mathrm{df}=1, \mathrm{P}=0.346\right)$. Similar mean counts $\left(1.99 \times 10^{3} \mathrm{CFU} / \mathrm{mL}\right)$ were observed in wells with an elevation 0.1-0.59 $\mathrm{m}$ and those with casing not raised. However, higher mean counts $(2.15 \times$ $10^{3} \mathrm{CFU} / \mathrm{ml}$ ) were obtained in wells with casing raised above $0.6 \mathrm{~m}$ (Table 4). The difference in total coliform counts between wells with different elevations was not significant $\left(X^{2}=0.658, \mathrm{df}=2, \mathrm{P}=0.720\right)$. A weak positive correlation $(+0.041)$ was observed between total coliform count and well elevation. Wells located within a distance of $10 \mathrm{~m}$ from sanitary structure had the highest mean counts $\left(2.24 \times 10^{3} \mathrm{CFU} / \mathrm{mL}\right)$ of total coliforms. There

Table 4 Relationship between well characteristics and total coliform counts of samples

\begin{tabular}{|c|c|c|c|c|c|c|}
\hline Characteristic & Category & $\begin{array}{l}\text { Mean counts } \\
(\mathrm{CFU} / \mathrm{ml})\end{array}$ & SE Mean & StDev & $\begin{array}{l}\text { Range } \\
\text { (CFU/ml) }\end{array}$ & $\begin{array}{l}\text { Correlation coefficient } \\
\text { ( } P \text { value })\end{array}$ \\
\hline \multirow[t]{2}{*}{ Condition of well aperture } & Covered & $1.98 \times 10^{3}$ & 186 & 2651 & $0-1.1 \times 10^{4}$ & \\
\hline & Not covered & $2.14 \times 10^{3}$ & 163 & 2561 & $0-1.4 \times 10^{4}$ & \\
\hline \multirow[t]{3}{*}{ Height of well casing above the ground $(\mathrm{m})$} & 0.00 & 1. $99 \times 10^{3}$ & 536 & 2783 & $2.0 \times 10^{1}-1.10 \times 10^{4}$ & $+0.041(0.205)$ \\
\hline & $0.1-0.59$ & $1.99 \times 10^{3}$ & 184 & 2654 & $0-1.40 \times 10^{4}$ & \\
\hline & $\geq 0.60$ & $2.15 \times 10^{3}$ & 172 & 2534 & $0-1.05 \times 10^{4}$ & $-0.154(0.000)$ \\
\hline \multirow[t]{3}{*}{ Distance between well and sanitary structure (m) } & $1.0-10.00$ & $2.24 \times 10^{3}$ & 148 & 2658 & $0-1.40 \times 10^{4}$ & \\
\hline & $10.1-15.00$ & $1.59 \times 10^{3}$ & 226 & 2352 & $0-1.10 \times 10^{4}$ & \\
\hline & $\geq 15.00$ & $1.73 \times 10^{3}$ & 642 & 2723 & $6.0 \times 10^{1}-9.0 \times 10^{4}$ & \\
\hline \multirow[t]{3}{*}{ State of well } & New & $2.07 \times 10^{3}$ & 473 & 2458 & $2.0 \times 10^{1}-8.9 \times 10^{3}$ & \\
\hline & Fairly old & $1.58 \times 10^{3}$ & 189 & 2200 & $0-1.10 \times 10^{4}$ & \\
\hline & Old & $2.31 \times 10^{3}$ & 164 & 2769 & $0-1.40 \times 10^{4}$ & \\
\hline \multirow[t]{2}{*}{ Presence of molded casing } & No & $2.03 \times 10^{3}$ & 262 & 2483 & $2.0 \times 10^{1}-1.40 \times 10^{4}$ & \\
\hline & Yes & $2.09 \times 10^{3}$ & 140 & 2641 & $0-1.10 \times 10^{3}$ & \\
\hline
\end{tabular}


was no significant difference in coliform counts with respect to distances from sanitary structure $\left(\chi^{2}=0.568, \mathrm{df}\right.$ $=2, \mathrm{P}=0.753)$. A negative correlation $(-0.154)$ occurred between well distance and total coliform counts. Mean counts were highest in old wells $\left(2.13 \times 10^{3} \mathrm{CFU} / \mathrm{mL}\right)$ (Table 4). The difference in counts with respect to state of wells was not significant $\left(x^{2}=3.105, \mathrm{df}=2, \mathrm{P}=0.212\right)$. The difference in total coliform counts between wells with molded casing (mean count $=2.09 \times 10^{3} \mathrm{CFU} / \mathrm{ml}$ ) and those without (mean count $=2.03 \times 10^{3} \mathrm{CFU} / \mathrm{mL}$ ) was also not significant $\left(X^{2}=2.579, \mathrm{df}=1, \mathrm{P}=0.108\right)$.

Fecal coliform counts were significantly higher $\left(\chi^{2}=\right.$ 4.202, $\mathrm{df}=1, \mathrm{P}=0.040$ ) in open wells (mean $=1.18 \times$ $10^{3} \mathrm{CFU} / \mathrm{mL}$ ) than in wells with a lid (mean $=1.15 \times$ $10^{3} \mathrm{CFU} / \mathrm{mL}$ ) (Table 5). Wells with casing not raised above ground $(0.00 \mathrm{~m})$ had the lowest mean count $(1.01 \times$ $10^{3} \mathrm{CFU} / \mathrm{ml}$ ) (Table 5). There was no significant difference in counts with respect to well casing elevation $\left(\chi^{2}=3.727\right.$, $\mathrm{df}=2, \mathrm{P}=0.155)$. A weak positive correlation $(+0.036)$ was observed between fecal coliform count and well elevation. This was not significant $(P=0.260)$. Counts decreased with increase in distance of well from sanitary structure. Wells located at a distance greater than $15 \mathrm{~m}$ had the lowest counts $\left(\right.$ mean $\left.=7.18 \times 10^{2} \mathrm{CFU} / \mathrm{mL}\right)$ (Table 5). However, the difference in counts with respect to distance was not significant $\left(X^{2}=0.794, \mathrm{df}=1, \mathrm{P}=0.672\right)$. Fecal coliform counts negatively correlated $(-0.131)$ with well distance from sanitary structure. There was no significant difference in fecal coliform count with respect to state of well $\left(\chi^{2}=\right.$ 1.193, $\mathrm{df}=2, \mathrm{P}=0.551$ ). Higher mean counts were found in wells with molded casing $\left(1.19 \times 10^{3} \mathrm{CFU} / \mathrm{ml}\right)$ than those without $\left(1.06 \times 10^{3} \mathrm{CFU} / \mathrm{mL}\right)$ (Table 5$)$ but the difference was not significant $\left(X^{2}=0.925, \mathrm{df}=1, \mathrm{P}=0.336\right)$.
Vibrio counts were higher in open wells (mean = $9.42 \times 10^{2} \mathrm{CFU} / \mathrm{ml}$ ) than in wells with a cover (mean = $\left.7.19 \times 10^{2} . \mathrm{CFU} / \mathrm{mL}\right)($ Table 6$)$. The difference was significant $\left(X^{2}=9.574, \mathrm{df}=1, \mathrm{P}=0.002\right)$. Wells with casing not raised above the ground recorded the highest Vibrio counts $\left(\right.$ mean $=1.0 \times 10^{3}$. CFU/mL). The lowest counts were from wells with an elevation greater than $0.6 \mathrm{~m}$ (Table 6). There was no significant difference in counts with respect to height of well casing $\left(X^{2}=0.767, \mathrm{df}=2\right.$, $\mathrm{P}=0.682)$. Vibrio counts negatively correlated $(-0.023)$ with well casing elevation. Similar to other bacteriologic parameters, Vibrio counts decreased with increase in distance from sanitary structure, with lowest counts recorded in wells located $\geq 15 \mathrm{~m}$ (Table 6). There was no significant difference in counts with respect to distance from sanitary structure $\left(x^{2}=2.852, \mathrm{df}=2, \mathrm{P}=0.240\right)$. Counts negatively correlated $(-0.157)$ with distance from sanitary structure. Higher mean counts were recorded in old wells $\left(9.50 \times 10^{2} \mathrm{CFU} / \mathrm{mL}\right)$ (Table 6). The difference was significant $\left(\mathrm{X}^{2}=15.435, \mathrm{df}=2, \mathrm{P}<\right.$ 0.001). The difference between counts in wells with molded casing and those without was not significant $\left(X^{2}=0.387, \mathrm{df}=1, \mathrm{P}=0.534\right)$.

\section{Identification of isolates}

A total of 936 bacteria isolated from samples were classified into 13 species based on their cultural, morphological and biochemical characteristics. Staphylococcus aureus (17.8\%), Escherichia coli (12.6\%) and Aeromonas hydrophila (10.4\%) were the most frequently isolated organisms while Vibrio cholerae (2.01\%) was the least (Table 7). V. cholerae isolates belonged to the non-O1 serogroup. In New Bell, isolates most frequently detected

Table 5 Relationship between well characteristics and fecal coliform counts of samples

\begin{tabular}{|c|c|c|c|c|c|c|}
\hline Characteristic & Category & $\begin{array}{l}\text { Mean counts } \\
(\mathrm{CFU} / \mathrm{ml})\end{array}$ & SE Mean & St Dev & $\begin{array}{l}\text { Range } \\
\text { (CFU/ml) }\end{array}$ & $\begin{array}{c}\text { Correlation coefficient } \\
\text { ( } P \text { value) }\end{array}$ \\
\hline \multirow[t]{2}{*}{ Condition of well aperture } & Covered & $1.15 \times 10^{3}$ & 132 & 1883 & $0-9.0 \times 10^{3}$ & \\
\hline & Not covered & $1.18 \times 10^{3}$ & 110 & 1722 & $0-9.0 \times 10^{3}$ & \\
\hline \multirow[t]{3}{*}{ Height of well casing above the ground (m) } & 0.00 & $1.01 \times 10^{3}$ & 332 & 1726 & $2.0-7.0 \times 10^{3}$ & $+0.036(0.260)$ \\
\hline & $0.1-0.59$ & $1.13 \times 10^{3}$ & 126 & 1807 & $0-9.0 \times 10^{3}$ & \\
\hline & $\geq 0.60$ & $1.23 \times 10^{3}$ & 122 & 1797 & $0-9.0 \times 10^{3}$ & \\
\hline \multirow[t]{3}{*}{ Distance between well and sanitary structure (m) } & $1.0-10.00$ & $1.27 \times 10^{3}$ & 103 & 1851 & $0-9.0 \times 10^{3}$ & $+0.131(0.000)$ \\
\hline & $10.1-15.00$ & $9.41 \times 10^{2}$ & 159 & 1649 & $0-9.0 \times 10^{3}$ & \\
\hline & $\geq 15.00$ & $7.18 \times 10^{2}$ & 346 & 1468 & $0-5.5 \times 10^{3}$ & \\
\hline \multirow[t]{3}{*}{ State of well } & New & $1.30 \times 10^{3}$ & 383 & 1990 & $0-8.0 \times 10^{3}$ & \\
\hline & Fairly old & $8.53 \times 10^{2}$ & 132 & 1539 & $0-9.0 \times 10^{3}$ & \\
\hline & Old & $1.30 \times 10^{3}$ & 111 & 1880 & $0-9.0 \times 10^{3}$ & \\
\hline \multirow[t]{2}{*}{ Presence of molded casing } & No & $1.06 \times 10^{3}$ & 190 & 1805 & $0-9.0 \times 10^{3}$ & \\
\hline & Yes & $1.19 \times 10^{3}$ & 95 & 1800 & $0-9.0 \times 10^{3}$ & \\
\hline
\end{tabular}


Table 6 Relationship between well characteristics and Vibrio counts of samples

\begin{tabular}{|c|c|c|c|c|c|c|}
\hline Characteristic & Category & $\begin{array}{l}\text { Mean counts } \\
\text { (CFU/ml) }\end{array}$ & SE Mean & StDev & $\begin{array}{l}\text { Range } \\
\text { (CFU/ml) }\end{array}$ & $\begin{array}{l}\text { Correlation coefficient } \\
\text { (P value) }\end{array}$ \\
\hline \multirow[t]{2}{*}{ Condition of well aperture } & Covered & $7.19 \times 10^{2}$ & 82 & 1171 & $0-8.0 \times 10^{3}$ & \\
\hline & Not covered & $9.42 \times 10^{2}$ & 81 & 1267 & $0-7.6 \times 10^{3}$ & \\
\hline \multirow[t]{3}{*}{ Height of well casing above the ground $(\mathrm{m})$} & 0.00 & $1.00 \times 10^{3}$ & 274 & 1424 & $0-6.60 \times 10^{3}$ & $-0.023(0.483)$ \\
\hline & $0.1-0.59$ & $8.58 \times 10^{2}$ & 92 & 1322 & $0-8.00 \times 10^{3}$ & \\
\hline & $\geq 0.60$ & $8.04 \times 10^{2}$ & 75 & 1107 & $0-7.20 \times 10^{3}$ & \\
\hline \multirow[t]{3}{*}{ Distance between wells and sanitary structure $(\mathrm{m})$} & $1.0-10.00$ & $9.18 \times 10^{2}$ & 70 & 1262 & $0-7.6 \times 10^{3}$ & $-0.157(0.000)$ \\
\hline & $10.1-15.00$ & $6.80 \times 10^{2}$ & 114 & 1186 & $0-8.0 \times 10^{3}$ & \\
\hline & $\geq 15.00$ & $4.16 \times 10^{2}$ & 119 & 506 & $3.0 \times 10^{1}-1.6 \times 10^{3}$ & \\
\hline \multirow[t]{3}{*}{ State of well } & New & $6.09 \times 10^{2}$ & 173 & 899 & $0-3.0 \times 10^{3}$ & \\
\hline & Fairly old & $6.69 \times 10^{2}$ & 97 & 1125 & $0-7.0 \times 10^{3}$ & \\
\hline & Old & $9.50 \times 10^{2}$ & 77 & 1295 & $0-8.0 \times 10^{3}$ & \\
\hline \multirow[t]{2}{*}{ Presence of molded casing } & No & $8.78 \times 10^{2}$ & 134 & 1271 & $0-8.0 \times 10^{3}$ & \\
\hline & Yes & $8.36 \times 10^{2}$ & 65 & 1222 & $0-7.20 \times 10^{3}$ & \\
\hline
\end{tabular}

Well aperture $\left(X^{2}=9.574, d f=1, P=0.002\right)$.Well height: TVC $\left(x^{2}=0.767, d f=2, P=0.682\right)$.

Distance well-pit latrine: TVC $\left(X^{2}=2.852, d f=2, P=0.240\right)$. State of well $\left(X^{2}=15.435, d f=2, P=0.000\right)$. Well casing $\left(X^{2}=0.387, d f=1, P=0.534\right)$.

were S. aureus (8.4\%), E. coli (8.2\%) and A. hydrophila (5.8\%). Predominant isolates in Bepanda included S. aureus (9.5\%), Citrobacter freundii (5.4\%) and A. hydrophila $(4.7 \%)$.

\section{Antibiotic sensitivity of isolates}

Ten of each of the 13 bacterial species isolated (total 130 isolates) were randomly selected and their susceptibility to antibiotics tested. Ciprofloxacin (96.2\%) was the most active antibiotic. Other drugs with high potency were

Table 7 Frequency of isolation of organisms from study sites

\begin{tabular}{llll}
\hline Isolate & Bepanda N (\%) & New bell N (\%) & Total N (\%) \\
\hline S. aureus & $89(9.5 \%)$ & $78(8.4 \%)$ & $167(17.8 \%)$ \\
E. coli & $41(4.4 \%)$ & $77(8.2 \%)$ & $118(12.6 \%)$ \\
A. hydrophila & $44(4.7 \%)$ & $54(5.8 \%)$ & $98(10.4 \%)$ \\
C. freundii & $51(5.4 \%)$ & $37(3.9 \%)$ & $88(9.4 \%)$ \\
P. aeruginosa & $42(4.5 \%)$ & $43(4.6 \%)$ & $85(9.1 \%)$ \\
E. aerogenes & $39(4.2 \%)$ & $32(3.4 \%)$ & $71(7.6 \%)$ \\
K. pneumoniae & $30(3.2 \%)$ & $38(4.1 \%)$ & $68(7.2 \%)$ \\
S. epidermidis & $31(3.3 \%)$ & $35(3.7 \%)$ & $66(7.0 \%)$ \\
V. mimicus & $22(2.3 \%)$ & $23(2.5 \%)$ & $45(4.8 \%)$ \\
Salmonella spp & $14(1.5 \%)$ & $28(3.0 \%)$ & $42(4.5 \%)$ \\
V. fluvialis & $20(2.1 \%)$ & $18(1.9 \%)$ & $38(4.1 \%)$ \\
V. vulnificus & $17(1.8 \%)$ & $17(1.8 \%)$ & $34(3.6 \%)$ \\
V. cholerae & $10(1.1 \%)$ & $9(1.0 \%)$ & $19(2.01 \%)$ \\
Total & $\mathbf{4 5 0 ( 4 7 . 9 \% )}$ & $\mathbf{4 8 9}(\mathbf{5 2 . 1} \%)$ & $\mathbf{9 3 9}(\mathbf{1 0 0 \% )}$ \\
\hline X. & &
\end{tabular}

$\left(\mathrm{X}^{2}=25.266, \mathrm{df}=14, \mathrm{P}=0.032\right) . \boldsymbol{N}$ number of isolates, \% percentage. gentamicin (88.5\%), tetracycline (76.9\%) and ceftriaxone (76.9\%). Ampicillin (19.2\%) and cotrimoxazole (34.6\%) showed low susceptibilities (Table 8). Forty-four $(33.8 \%)$ isolates were multidrug resistant (resistant to 3 or more antibiotics) with Salmonella spp showing two resistance patterns $D^{2} X^{R} C^{2} T^{R} T^{R} A M P^{R} C E F^{R}$ and $\mathrm{DOX}^{\mathrm{R}} \mathrm{COT}^{\mathrm{R}} \mathrm{TE}^{\mathrm{R}} \mathrm{AMP}^{\mathrm{R}} \mathrm{CHL}^{\mathrm{R}}$ (Table 9). All Samonella spp, E. coli and Pseudomonas aeruginosa tested were multidrug resistant and showed resistance to ampicillin and/or ceftriaxone.

MICs were determined for isolates that were not multidrug resistant. MIC values varied with agent and the organism tested. All isolates were sensitive to the various antibiotics with the exception of Staphylococcus aureus and Staphylococcus epidermidis which had intermediate sensitivity to tetracycline $(\mathrm{MIC}=7.5 \mu \mathrm{g} / \mathrm{ml}$ for each of the organisms) and gentamicin ( $\mathrm{MIC}=5 \mu \mathrm{g} / \mathrm{ml}$ each) (Table 10). Lowest MIC values were obtained for ciprofloxacin $(0.12-0.938 \mu \mathrm{g} / \mathrm{ml})$, followed by gentamicin (on Klebsiella pneumoniae and Enterobacter aerogenes) $(0.313 \mu \mathrm{g} / \mathrm{ml}$ each)). V. mimicus, $V$. vulnificus, A. hydrophila and $K$. pneumoniae showed low MIC to ceftriaxone. The staphylococci had the highest MIC for all drugs tested.

\section{Extended spectrum $\beta$-lactamase production potential of resistant isolates}

Sixty (60) multi-drug resistant isolates were screened for extended spectrum $\beta$-lactamase (ESBL) production potential. Thirty (50\%) of isolates demonstrated the potential for ESBL production (Table 11). All Salmonella spp, C. fruendii and $E$. coli exhibited the potential for extended spectrum $\beta$-lactamase production. 
Table 8 Antibiotic susceptibility (\%) of isolates

\begin{tabular}{lcccccccc}
\hline Isolates & TE & CHL & CIP & AMP & CEF & SXT & GEN & DXT \\
\hline A. Hydrophila & 100 & 60 & 100 & 0 & 100 & 0 & 100 & 100 \\
C. freundii & 100 & 0 & 100 & 0 & 0 & 0 & 100 & 100 \\
E. aerogenes & 100 & 100 & 100 & 40 & 100 & 100 & 100 & 0 \\
E. coli & 100 & 0 & 50 & 0 & 0 & 0 & 100 & 0 \\
K. pneumoniae & 100 & 100 & 100 & 0 & 100 & 100 & 100 & 0 \\
P. aeruginosa & 0 & 100 & 100 & 0 & 100 & 0 & 100 & 0 \\
S. aureus & 100 & 100 & 100 & 100 & 100 & 100 & 100 & 100 \\
S. epidermidis & 100 & 100 & 100 & 100 & 100 & 100 & 100 & 100 \\
Salmonella spp & 0 & 50 & 100 & 0 & 0 & 0 & 100 & 0 \\
V. cholerae & 100 & 0 & 100 & 0 & 100 & 50 & 0 & 100 \\
V. fluvialis & 0 & 100 & 100 & 0 & 100 & 0 & 100 & 100 \\
V. mimicus & 100 & 100 & 100 & 0 & 100 & 0 & 100 & 100 \\
V. vulnificus & 100 & 100 & 100 & 0 & 100 & 0 & 100 & 100 \\
Total & $\mathbf{7 6 . 9}$ & $\mathbf{7 0}$ & $\mathbf{9 6 . 2}$ & $\mathbf{1 9 . 2}$ & $\mathbf{7 6 . 9}$ & $\mathbf{3 4 . 6}$ & $\mathbf{8 8 . 5}$ & $\mathbf{6 3 . 1}$
\end{tabular}

TE tetracycline, CHL chloramphenicol, CIP ciprofloxacin, AMP ampicillin, CEF ceftriaxone, SXT cotrimoxazole, GEN gentamicin, DXT doxycycline.

\section{Discussion}

In developing countries increase in human population and industrialization has exerted an enormous pressure on the provision of safe drinking water. Provision of high quality water as well as protecting and conserving scarce water resources is therefore one of the greatest challenges currently facing national and regional governments [21]. We report here the factors affecting the bacteriological quality of well water in New Bell and Bepanda in Douala, Cameroon. This information will strengthen efforts in the provision of safe water to inhabitants of study sites.

Total viable bacterial counts $\left(0-4.25 \times 10^{4} \mathrm{CFU} / \mathrm{mL}\right)$, total coliform counts $\left(0-1.4 \times 10^{4} \mathrm{CFU} / \mathrm{mL}\right)$, fecal coliform $\left(0-9.0 \times 10^{3} \mathrm{CFU} / \mathrm{mL}\right)$ and Vibrio counts $(0-8.0 \times$ $10^{3} \mathrm{CFU} / \mathrm{mL}$ ) were high throughout the period of study (Table 1). Of the 150 wells sampled, one well (in Bepanda) recorded no bacterial contamination (0 CFU/ $\mathrm{mL}$ ) throughout the study period. In New Bell, bacterial
Table $10 \mathrm{MIC}(\mu \mathrm{g} / \mathrm{ml})$ of active antibiotics to isolates tested

\begin{tabular}{|c|c|c|c|c|c|c|c|c|}
\hline Isolate & DOX & СOT & CIP & TE & AMP & GEN & CEF & $\mathrm{CHL}$ \\
\hline S. aureus & 3.75 & 12.5 & 0.938 & 7.5 & 5 & 5 & 7.5 & 7.5 \\
\hline S. epidermidis & 3.75 & 12.5 & 0.938 & 7.5 & 5 & 5 & 7.5 & 7.5 \\
\hline E. aerogenes & ND & 6.25 & 0.47 & 0.47 & ND & 0.313 & 1.88 & 7.5 \\
\hline K. pneumoniae & ND & 3.13 & 0.12 & 0.94 & ND & 0.313 & 0.94 & 7.5 \\
\hline V. mimicus & 3.75 & ND & 0.23 & 0.47 & ND & 5 & 0.94 & 7.5 \\
\hline V. vulnificus & 3.75 & ND & 0.117 & 0.94 & ND & 5 & 0.47 & 7.5 \\
\hline A. hydrophila & 3.75 & ND & 0.47 & 0.94 & ND & 5 & 0.47 & 7.5 \\
\hline
\end{tabular}

counts of $0 \mathrm{CFU} / \mathrm{mL}$ were obtained from one well only during the dry season. These wells recording $0 \mathrm{CFU} / \mathrm{ml}$ were disinfected by chlorination and used as a source of drinking water. In New Bell, chlorination was abandoned during the rainy season and bacterial contamination was detected in water. Samples from other wells were heavily contaminated and did not meet the WHO [22] standards that stated that coliforms or fecal coliform must not be detectable in any $100 \mathrm{~mL}$ of drinking water. TVBC exceeded the EPA [23] limit of $1.0 \times 10^{2} \mathrm{CFU} / \mathrm{mL}$. These findings are worrisome. Uses of well water reported included laundry and washing of kitchen utencils (100\%), cooking (73\%) and washing of fruits and vegetables (50.4\%) (Additional file 1). These could predispose to waterborne infections. High counts of indicator bacteria suggest heavy pollution of water with fecal matter. Recent studies in other developing countries [24,25] have also reported high TVBC, total coliform and fecal coliforms in ground water. Significant differences $(P<0.05)$ in bacterial counts were observed between seasons, with higher counts recorded in the rainy season than in the dry season (Table 2). The city of Douala is poorly drained. During heavy rains, most parts of the study area are flooded and this could result in well contamination. Rainfall and flooding events have been shown to

Table 9 Resistance patterns of isolates

\begin{tabular}{|c|c|c|c|}
\hline Isolate & Resistance pattern & $\begin{array}{l}\text { Number of isolates } \\
\text { with resistance pattern }\end{array}$ & $\begin{array}{l}\text { Percentage (\%) of isolates } \\
\text { showing resistance pattern }\end{array}$ \\
\hline P. aeruginosa & DOX $^{R}$ COT $^{R} T^{R}{ }^{R} A M P^{R}$ & 10 & 100 \\
\hline \multirow[t]{2}{*}{ Salmonella spp } & $D O X^{R} C O T^{R} T^{R} A M P^{R} C_{E F}^{R}$ & 5 & 50 \\
\hline & DOX ${ }^{R} C^{R} T^{R} T^{R} A M P^{R} C^{2} F^{R} C H L^{R}$ & 5 & 50 \\
\hline V. fluvialis & $\mathrm{COT}^{R} T^{R} \mathrm{AMP}^{\mathrm{R}}$ & 4 & 40 \\
\hline C. freundii & $\mathrm{COT}^{\mathrm{R}} \mathrm{AM} \mathrm{P}^{\mathrm{R}} \mathrm{CEF}^{\mathrm{R}} \mathrm{CHL} \mathrm{L}^{\mathrm{R}}$ & 5 & 50 \\
\hline E. coli & DOX $^{R}$ COT $^{R}$ AMP $^{R} \mathrm{CEF}^{R} \mathrm{CHL}^{\mathrm{R}}$ & 10 & 100 \\
\hline V. cholerae & $\mathrm{COT}^{R} \mathrm{AMP}^{R} \mathrm{GEN}^{R} \mathrm{CHL}^{\mathrm{R}}$ & 5 & 50 \\
\hline Total & & 44 & 33.8 \\
\hline
\end{tabular}

TE tetracycline, CHL chloramphenicol, CIP ciprofloxacin, AMP ampicillin, CEF ceftriaxone, SXT cotrimoxazole, GEN gentamicin, DXT doxycycline. 


\begin{tabular}{|c|c|c|c|}
\hline Isolate & Number tested & $\begin{array}{l}\text { Number } \\
\text { positive (\%) }\end{array}$ & $\begin{array}{l}\text { Number } \\
\text { negative (\%) }\end{array}$ \\
\hline P. aeruginosa & 10 & 0 & $10(100 \%)$ \\
\hline Salmonella spp & 10 & $10(100 \%)$ & 0 \\
\hline C. freundii & 10 & $10(100 \%)$ & 0 \\
\hline V. cholerae & 10 & 0 & $10(100 \%)$ \\
\hline V. fluvialis & 10 & 0 & 10 (100\%) \\
\hline E. coli & 10 & $10(100 \%)$ & 0 \\
\hline Total & 60 & $30(50 \%)$ & $30(50 \%)$ \\
\hline
\end{tabular}

negatively impact well water quality [26] resulting in outbreaks of waterborne diseases as flood water carry microbial contaminants into unprotected water sources. Inhabitants dispose domestic waste in pits (29.3\%), public vats $(64.2 \%)$ and in the open (6.5\%). Public vats at times are not regularly emptied when full. Under such circumstances, continuous dumping of wastes results in overflow to the surrounding. During heavy rains, these wastes could be washed into water sources contributing to pollution. A strong positive correlation occurred between fecal colifoms and total colifom counts $(+0.635)$, but correlation between total coliforms and TVBC $(+0.468)$, fecal coliform counts and Vibrio counts $(+0.433)$ though positive was weaker. This finding suggests that these organisms could have originated from same or similar sources of contamination.

Well characteristics including construction and site management have been shown to be the most important factors responsible for well vulnerability to contamination [27]. We evaluated the relationship between well characteristics and bacteriological quality of well water. Total viable bacterial counts (Table 3), total coliform counts (Table 4), fecal coliform counts (Table 5) and Vibrio counts (Table 6) were higher in open wells than in wells with a lid. High bacterial counts also occurred in wells irrespective of height of casing above ground (Tables 3, 4, 5 and 6). A weak negative correlation occurred between well casing elevation and TVBC $(-0.003)$ and also with Vibrio counts $(-0.023)$. This shows that increasing well height will result in only a minor decrease in these counts. However, a weak positive correlation was observed between elevation and total coliform counts $(+0.041)$ (Table 4) and also with fecal coliform counts $(+0.036)$ (Table 5). This was not significant $(\mathrm{P}>0.05)$. Thus counts of these bacteria in well water will increase only slightly with increase in well height. Bacterial counts decreased with increase in well distance from sanitary structure (Tables 3, 4, 5 and 6). A negative correlation was seen between well distance and TVBC $(-0.119)$, total coliform counts (-0.154), fecal coliforms (-0.131) and, Vibrio counts $(-0.121)$ confirming the fact that increasing the distance between wells and sanitary structure would reduce water contamination. Although respondents demonstrated a good knowledge of waterborne diseases (100\%), well water contamination and possible sources of contamination (76.5\%) (Additional file 1), an indication of the success of the sensitization campaigns by the Ministry of Public Health, wells were not protected. Majority of wells were not constructed following recommended guidelines of $0.6 \mathrm{~m}$ well casing above ground [28] and a lateral separation distance of at least $30 \mathrm{~m}$ from sanitary facility [29]. Eighty-two (54.7\%) wells were not covered, 52\% had well casing $<0.6 \mathrm{~m}$ above ground and $96 \%$ wells were located at a distance $<15 \mathrm{~m}$ from sanitary structure (Additional file 1). These factors may increase the risk of contamination and account for our observations. Findings of our study show that even protected wells were still exposed to contamination. Covered wells with casing raised above $0.6 \mathrm{~m}$ which were poorly maintained, located near sanitary structures were subject to contamination. This may explain our finding of no significant difference in counts between covered and open wells and between wells with different casing elevations. Use of pit latrines (93\%) is common in study sites. The distance between wells and sanitary structure ranged from 1 to $17.4 \mathrm{~m}$. Overcrowding in study sites leaves inhabitants with limited distance between wells and sanitary structures. The close proximity of wells to sanitary infrastructure (mean distance of $7.4 \mathrm{~m}$ and $7.6 \mathrm{~m}$ in Bepanda and New Bell respectively) in addition to the presence of cracks in most casings could facilitate seepage from pit latrines contaminating well water. Wells in study sites are shallow [8] and could also be easily contaminated through seepage from latrines through the sandy, porous soil in Douala. However, to confirm this, studies focusing only on protected wells need to be carried out. Thus, poor town planning, indiscriminate well siting, poor maintenance and construction of wells may account for the poor water quality in study sites. Muruka et al. [30] detected an indirect association between well distance from pit latrines and fecal contaminants, with a decrease of 3.38 fecal coliforms $/ 100 \mathrm{~mL}$ counts for every $1 \mathrm{~m}$ increase in distance. Asheesh [31] did not find any association between dugwell bacteriological quality and distance to the nearest pit latrines contradicting our findings. However, similar to our findings, Adetunji and Odetokun [24] reported a negative correlation between well distance and aerobic bacterial counts. Thus, as long as these wells and sanitary structures are in close proximity, the potential health hazard posed by highly polluted water cannot be overemphasized especially as $73 \%$ of respondents reported to have been victims of waterborne disease.

Inhabitants (100\%) used the bucket and rope method to lift water from wells. This method is cheaper but could introduce contaminants into water. The use of a submersible pump is recommended but is unaffordable due to 
restricted financial resources of inhabitants. Although wells were disinfected, $88.5 \%$ of respondents used table salt as disinfectant and frequency of disinfection was irregular (Additional file 1). This is inappropriate and explains the heavy microbial contamination recorded in wells. Well characteristics and hygiene and sanitary practices in study sites were similar, hence there were no significant differences in bacteriological quality of water between sites.

Bacteria isolated included both pathogenic and potentially pathogenic species. Overall S. aureus (17.8\%), E. coli (12.6\%) and A. hydrophila (10.4\%) predominated. There was variation in predominant isolates with site (Table 7). S. aureus, a normal flora of the skin, is a well recognized pathogen. It has been associated with a large number of infections including food intoxication, community acquired urinary tract infections [32], conjunctivitis, scalded skin syndrome, toxic shock, respiratory infections and skin infections. E. coli (12.6\%) the second predominant isolate is a major public health concern as it not only indicates recent contamination with fecal matter and the possible presence of intestinal pathogens but due to the fact that certain pathogenic strains of the organism such as the enteropathogenic O157:H7 responsible for several out breaks of bloody diarrhea [33] have been detected in water. A. hydrophila (10.4\%) is an opportunistic pathogen particularly in immunocompromised individuals. They cause non-gastrointestinal infections in humans and have been isolated from drinking water even after chlorination [34]. Its presence in our samples as one of the predominant isolates is thus a cause for concern. Other enteric organisms isolated included Citrobacter fruendii (9.4\%), Enterobacter aerogenes (7.6\%), Klebsiella pneumoniae (7.2\%) and Salmonella spp (4.5\%). Their isolation further confirms the contamination of water with fecal material and the possible presence of other waterborne enteric pathogens such as viruses and protozoa not included in our study. These enteric isolates are also of public health importance as they have been associated with several infections. Pseudomonas aeruginosa $(9.1 \%)$ is an opportunistic pathogen responsible for a wide range of acute and chronic infections [35] when introduced into areas devoid of normal defenses. It is a major cause of bacteremia, soft tissue infections, conjunctivitis, infections of burns and wounds, cystic fibrosis, endocarditis and otitis media [36].

Four species of Vibrio were isolated from samples: Vibrio mimicus (4.8\%), V. fluvialis (4.1\%) V. vulnificus (3.6\%) and $V$. cholerae $(2.01 \%)$ (Table 7 ) all of which have been shown to cause infections in man and aquatic organisms. $V$. mimicus is closely related to $V$. cholerae in terms of pathogenesis of infections. Pathogenic strains cause cholera-like diarrhea in man [37] as well as infections in fish [38] and have been reported to express same virulence factors as $V$. cholerae [38]. $V$. fluvialis is an important cause of cholera-like bloody diarrhea in humans and is commonly found in areas with poor sanitation [39]. V. vulnificus has been shown to cause gastroenteritis and is the leading cause of reported death resulting from seafood consumption [40]. Serological typing of $V$. cholerae isolates showed that they belonged to the non-O1 serogroup. Although not responsible for epidemic cholera, the presence of the non-O1 serogroup in well water cannot be overlooked as they have been associated with diarrheal disease [41]. Certain strains have been shown to contain virulence factors present in $\mathrm{O} 1$ strain [42]. Furthermore, non-O1 serogroup have been reported to undergo serogroup conversion resulting in emergence of new strains with pathogenic potential [43]. Recent studies carried out in our study area [44] have reported the co-existence of $\mathrm{O} 1$ and non-O1 strains in well water.

It is generally believed that groundwater is relatively free of microorganisms and thus fit for consumption. However, findings from present study show that water from dug wells in New Bell and Bepanda contain high numbers of pathogenic enteric bacteria. Ndjama et al. [4] reported a high prevalence of waterborne diseases in other parts of Douala. Our findings together with the report of Ndjama et al. [4] demonstrate that waterborne diseases could be a significant health challenge in Douala. However, to determine the role of our isolates in causing diarrheal disease, their enterotoxin production potential has to be investigated. Although the Ministry of Public Health carries out sensitization campaigns on well disinfection particularly during cholera outbreaks, findings from our study show an urgent need for a regular and sustained disinfection programme in study sites.

Ciprofloxacin (96.2\%) was the most effective antibiotic among those tested. Other potent antibiotics included gentamicin $(88.5 \%)$, tetracycline $(76.9 \%)$, ceftriaxone $(76.9 \%)$, chloramphenicol (70\%) and doxycycline (63.1\%) (Table 8). Ciprofloxacin and gentamicin are relatively expensive drugs. In addition, gentamicin is formulated as injection. These factors discourage procurement and misuse of these drugs. Drug misuse particularly through automedication is a serious public health concern in Cameroon. Susceptibility to ampicillin (19.2\%), cotrimoxazole (34.6\%) and to a lesser extend doxycycline (63.1\%) was low. These drugs were heavily used for treatment and prophylaxis during past cholera outbreaks in Douala $[45,46]$ and could have resulted in selection of resistant bacteria. Considering that resistance markers have the potential of spreading across bacteria species, these organisms might have acquired resistance by various mechanisms. Forty-four (33.8\%) isolates were multidrug resistant (Table 9) with twenty (20) showing resistance to five or more drugs. All (100\%) $P$. aeruginosa, Salmonella spp, and E. coli tested were multi- 
drug resistant. Occurrence of multi-drug resistant bacteria in wells thus constitutes a health threat.

Ciprofloxacin recorded the lowest MIC values (0.117$0.938 \mu \mathrm{g} / \mathrm{ml}$ ) on all the isolates tested and among all the drugs (Table 10). With the exception of the staphylococci, which showed intermediate susceptibility to tetracycline and gentamicin (susceptibility break point = $8.0 \mu \mathrm{g} / \mathrm{ml}$ respectively), MIC determination confirmed isolates to be sensitive to antibiotics. These drugs are therefore of great value in eradication of waterborne infections in study sites. We did not use a control strain in antibiotic susceptibility testing. This constitutes a limitation to our study.

Observing that all multidrug resistant isolates were resistant to ampicillin and/or ceftriaxone, we tested isolates for extended spectrum beta-lactamase (ESBL) production potential as a possible mechanism of resistance to $\beta$ lactams. ESBL production was observed in all Salmonella species, Citrobacter fruendii and E. coli Talble 11. (This greatly limits:in treatment of infections caused by these pathogens. Our findings show that well water could play an important role in the dissemination of these ESBL producing organisms.

\section{Conclusion}

Poor water quality in study area could be due to inadequate hygiene and sanitation, well disinfection and well characteristics. Our findings show that even after experiencing several devastating outbreaks of cholera, waterborne diseases will continue to be a health problem in study sites if appropriate measures are not taken to improve on water quality.

\section{Recommendations}

There is need for enforcement of public laws on siting and construction of pit latrines, and guidelines for well construction. Distribution or subsidizing the cost of chlorine and creation of a permanent structure which ensures regular disinfection of wells and not only during disease outbreak is of utmost importance. Health and hygiene and sanitation education which should emphasize on adequate water disinfection should be carried out regularly in study area. A comprehensive study which includes the bacteriological and physico-chemical characteristics of dug-wells and tube wells needs to be carried out to provide a clear picture of ground water quality in study sites.

\section{Additional file}

Additional file 1: Responses from questionnaire. Description of data: This is a summary of responses from questionnaire administered to inhabitants of study sites to evaluate their knowledge on waterborne diseases, and their hygiene and sanitation practices.

\section{Abbreviations}

CDE: Camerounaise des eaux; FET: Fisher's exact test.

\section{Competing interests}

The authors declare that they have no competing interests.

\section{Authors' contributions}

JTKA as principal investigator conceived, designed and coordinated the study, interpreted data and initiated the writing of the manuscript. NBLO collected samples, isolated and characterized bacteria carried out antimicrobial susceptibility testing. MTN participated in sample collection and isolation and characterization of bacteria. All authors read and approved the final manuscript.

\section{Acknowledgements}

We thank the well owners and the administration of Douala Deuxieme and Douala Troisieme where study sites are located for granting authorization to collect samples from wells and also inhabitants interviewed. We are grateful to Mr. Tanyimoh Nembualum for his assistance in statistical analysis. This work was carried out in the Laboratory for Emerging Infectious Diseases at the University of Buea.

Received: 14 March 2013 Accepted: 29 July 2013

Published: 29 July 2013

\section{References}

1. Kimani-Murage EW, Ngindu AM: Quality of water the slum dwellers use: the case of a Kenyan slum. J Urban Health 2007, 84:829-838.

2. UNICEF: Progress for children. A Report Card on Water and Sanitation. No. 5 September 2006. :1-36.

3. Kuitcha D, Ndjama J, Tita AM, Lienou G, Kamgang KBV, Ateba BH, Ekodeck GE: Bacterial contamination of water points of the upper Mfoundi watershed, Yaounde, Cameroon. Afr J Microbiol Res 2010, 4(7):568-574.

4. Ndjama J, Kamgang KBV, Sigha NL, Ekodeck GE, Awah TM: Water supply, sanitation and health risks in Douala, Cameroon. Afr J Environ Sci Technol 2008, 2(12):422-429.

5. Katte $\mathrm{VY}$, Fonteh MF, Guemuh GN: Domestic water quality in urban center in Cameroon: a case study of Dschang in the West Province. Afr Water J 2003, 10:43-54.

6. Mpakam HG: Vulnérabilité à la pollution des ressources en eaux à Bafoussam et incidences socio-économiques et sanitaires : modalités d'assainissement. Doctor of Philosophy, University Yaoundé l; 2009:267.

7. Tita MA, Kamgang KBV, Tsala GN: Microbial pollution of surface water and its health impact in the Nkoup River Basin in Foumbot, West Cameroon. J Microbiol Res 2009, 8:52-57.

8. Guevart E, Noeske J, Essomba JM, Edjenguele M, Bita A, Mouangue A, Manga B: Factors contributing to endemic cholera in Douala, Cameroon. Medicine Tropicale 2006, 66(3):283-291.

9. Millenium Development Goals. http://en.wikipedia.org/wiki/Millennium_ Development_Goals

10. Zephania NF, Fombutio CN: Tropical city milieu and disease infection: The case of Douala, Cameroon. J Hum Ecol 2010, 30(2):123-130.

11. Ngandjio A, Tejiokem M, Wouafo M, Ndome I, Yonga M, Guenole A, Lemee L, Quilici M-L, Fonkoua M-C: Antimicrobial resistance and molecular characterization of Vibrio cholerae 01 during the 2004 and 2005 outbreak of cholera in Cameroon. Foodborne Pathog Dis 2009, 6(1):49-56.

12. National Institute of Statistics (NIS): Cameroon in figures. Yaounde: National Institute of Statistics (NIS); 2006.

13. Fombe LS, Balgah SN: The urbanisation process in Cameroon, Patterns, Implications and Prospects. New York: Nova Publishers Inc; 2010:212.

14. Mafany GT: Impact of the geology and seawater intrusion on groundwater quality in Douala. MSc. Thesis, Department of Geology and Environmental Science. University of Buea; 1999:89.

15. Siham N, Taha MH: Superficial bacterial contamination of ovine and bovine carcasses at El-Harrach slaughterhouse (Algeria). Eur J Sci Res 2009, 38(3):474-485.

16. Shanmugam $V$, Santhanam $R$ : Beneficial uses of probiotics in mass scale production of marine ornamental fish. Afr J Microbiol Res 2009, 3(4):185-190

17. Center for Disease Control and Prevention (CDC): Response to cholera outbreak in Haiti. 2010. http://www.cdc.gov/haiticholera/update/ 
18. Akoachere J-FTK, Ndip RN, Chenwi EB, Ndip LM, Njock TE, Anong DN: Antibacterial effect of Zingiber officinale and Garcinia kola on respiratory tract pathogens. East Afr Med J 2002, 79(11):588-592.

19. Sinha M, Srinivasa $H$, Macaden $R$ : Target modification as antibiotic resistance profile and extended spectrum beta-lactamase (ESBL) production in Acinetobacter species. Indian J Med Res 2007, 126(1):63-67.

20. Clinical and Laboratory Standards Institute: Performance standards for antimicrobial disk and dilution susceptibility test for bacteria isolated from animals. Approved standard-3rd Edition. M31-A314 2005, vol. 24 No 1.

21. Jamieson R, Gordon R, Joy D, Lee H: Assessing microbial pollution of rural surface waters: a review of current watershed late modeling approaches. J Agr Water Manag 2004, 70:1-17.

22. World Health Organization (WHO): Guidelines for Drinking Water Quality. 3rd Edition. Incorporating the First and Second Addenda, Recommendations, Volume 1. Geneva: WHO; 2008.

23. US Environmental Protection Agency (EPA): Safe Drinking Water Act Amendment; 2002. http://water.epa.gov/drink/index.cfm

24. Adetunji VO, Odetokun IA: Groundwater contamination in Agbowo community, Ibadan Nigeria: impact of septic tanks distances to well. Malayas J Microbiol 2011, 7(3):159-166.

25. Akinbile CO, Yussof MS: Environmental impact of leachate pollution on groundwater supplies in Akure, Nigeria. Int J Environ Sci Dev 2011, 2(1):81-86.

26. Stukel TA, Greenberg ER, Dain BJ, Reed FC, Jacobs NJ: A longitudinal study of rainfall and coliform contamination in small community drinking water supplies. Environ/ Sci Technol 1990, 24:571-575.

27. Valenzuela M, Lagos B, Claret M, Mondaca MA, Perez C, Parra O: Fecal contamination of groundwater in a small rural dry land watershed in central Chile. Chil J Agr Res 2009, 69(2):235-243.

28. Susan J, Susan LS, Fred M, Fred K: Reducing the risk of ground water contamination by improving drinking water well condition. In New Jersey Assessment System; 1995:1-176.

29. Stenstrom TA: Tracing bacteria from latrine to ground water dug-wells, Paper presented at the Conference on Safe water Environments, Eldoret, Kenya. 1995 August 21-23; 1995.

30. Muruka C, Fagbamigbe FA, Muruka A, Njuguna J, Otieno DO, Onyando J, Wanjiku ZS, Onyango Z: The relationship between bacteriological quality of dug-wells and, pit latrines siting in an unplanned peri-urban settlement: a case study of Langas-Eldoret Municipality, Western Kenya. Public Health Res 2012, 2(2):32-36.

31. Asheesh A: A longitudinal study of relationship between water quality, hygiene behavior and childhood diarrhea in Langas, Eldoret. Eldoret: Moi University Community-Based Education Service Programme (COBES) Publication; 1994:1-10.

32. Akoachere J-FKT, Suylika Y, Njom HA, Nkie SE: Etiologic profile and antibacterial susceptibility of community acquired urinary tract infection in two Cameroonian towns. BMC Res Notes. 2012, 5:219.

33. Geldreich EK, Fox JG, Rice RE, Swerdlow D: Searching for a water supply connection in the Cabool, Missouri Disease outbreak of Escherichia coli O157:H7. Water Res J 1992, 26:8.

34. Burke V, Robinson J, Gracey M, Peterson D, Partridge K: Isolation of Aeromonas hydrophila from a metropolitan water supply: seasonal correlation with clinical isolates. Appl Environ Microb 1984, 48:361-366.

35. Ruxana ST, Timothy BS, John CW, Alice PS: Pathogenic-host interaction in Pseudomonas aeruginosa in pneumonia. Am J Resp Critl Care 2005, 1:1-3.

36. Valle DR, Castilb BJM, Fernandez FMJ, Bueso SE, Casa MJM, Sanchez GJ: Bacteremia, meningitis and brain abscesses in a hospitalized infant: complications of Pseudomonas aeruginosa in conjunctivitis. J Perinatol 2007, 19(6):462-465.

37. Murad L, Decy SS, Periska T, Cyrus HS, Narain HR, James RC, Buhari O: Spectrum of Vibrio species associated with acute diarrhea in North Jakarta, Indonesia. Diagn Micr Infect Dis 2002, 42(2):91-97.

38. Chakraborty S, Nair GB, Shiinoda S: Pathogenic vibrios in the natural aquatic environment. Rev Environ Health 1997, 12(2):63-80.

39. Etinosa $O$, Anthony S: Vibrio fluvialis: an unusual enteric pathogen of increasing public concern. Int J Environ 2010, 7:3628-3643.

40. Hlady WG, Klontz KC: The epidermiology of Vibrio infections in Florida. J Infect Dis 1996, 173:1176-1183.

41. Dalsgaard AJ, Albert MJ, Taylor DN, Shimada T, Meza R, Serichantalergs O, Echeverria P: Characterization of Vibrio cholerae non-O1 serogroup obtained from an outbreak of diarrhea in Lima, Peru. $J$ Clin Microbiol 1995, 33(10):2715-2722.

42. Islam MS, Siddika A, Khan MNH, Goldar MM, Sadique MA, Kabir ANMH, Huq A, Colwell RR: Microbiological analysis of tube-well water in rural area of Bangladesh. Appl Environ Microb 2001, 67(7):3328-3330.

43. Shah M, Asadulgham MNS, Abdul ARM, John MA, Nasirul KM, Mekalanos $\mathrm{J}$ : Analysis of clinical and environmental strains of non-toxigenic Vibrio cholerae for susceptibility to CTXФ: Molecular basis for origination of new strains with epidemic potential. Infect Immun 1998, 66(12):5819-5825

44. Tatah AJ-FK, Pulcherie KMC, Mande NL, Akum NH: Investigation of water sources as reservoirs of Vibrio cholerae in Bepanda, Douala and determination of physico-chemical factors maintaining its endemicity. Onderstepoort J Vet Res 2012, 79(2):Art. \#484. doi:10.4102/ojvr.v79i2.484

45. Guévart E, Noeske J, Sollé J, Mouangue A, Bikoti JM: Large-scale selective antibiotic prophylaxis during the 2004 cholera outbreak in Douala (Cameroon). Sante 2007, 17(2):63-68.

46. Guévart E, Van HC, Noeske J, Sollé J, Bita FA, Manga B: Diffuseur artisanal de chlore pour désinfecter les puits lors de l'épidémie de choléra de Douala. Medicine Tropicale 2008, 68:507-513.

\section{doi:10.1186/1471-2458-13-692}

Cite this article as: Akoachere et al:: Assessment of the relationship between bacteriological quality of dug-wells, hygiene behaviour and well characteristics in two cholera endemic localities in Douala, Cameroon. BMC Public Health 2013 13:692.

\section{Submit your next manuscript to BioMed Central and take full advantage of:}

- Convenient online submission

- Thorough peer review

- No space constraints or color figure charges

- Immediate publication on acceptance

- Inclusion in PubMed, CAS, Scopus and Google Scholar

- Research which is freely available for redistribution 